\title{
Choice of Posterior Subtemporal Transtentorial Approach for Tumor Resection in Deep Anteromedial Superior Cerebellum
}

\author{
-Case Report- \\ Takaaki BePPU*,**, Ryonoshin HIROOKA*, Shunrou FUjIWARA*, \\ Hiroshi KASHIMURA*, Hideaki NISHIMOTO*, \\ Kuniaki OGASAWARA*, and Akira OGAWA* \\ ${ }^{*}$ Department of Neurosurgery, and \\ ${ }^{* *}$ Division of Hyperbaric Medicine, Iwate Medical University, Morioka, Iwate
}

\begin{abstract}
The anteromedial superior cerebellar tumor can be accessed by various routes. For tumor presenting at the cerebellar surface in this region, the optimal approach remains contentious. Furthermore, which of the various routes offers the optimal approach to a tumor that is not present at the cerebellar surface but lies deep anteromedial superior cerebellum is a matter of debate. We report herein the case of a 44-yearold woman with hemangioblastoma deep within the subcortex of the anteromedial superior cerebellum. Preoperative magnetic resonance (MR) imaging and three-dimensional anisotropy contrast MR axonography using diffusion-weighted MR imaging demonstrated that the posterior subtemporal transtentorial (PSTT) approach would provide a shorter surgical corridor, minimal cerebellar split, and better preservation of nerve fibers, compared to the other approaches. Surgical tumor removal was successfully achieved using the PSTT approach. During surgery, the PSTT approach provided an operative field that enabled visualization of the proximal side of the superior cerebellar artery as the tumor feeding vessel. Although the vein of Labbé inserted just into the transverse-sigmoid junction, injury to this vein was avoided using optimal head position, cerebrospinal fluid drainage, and various devices. For patients with tumor located within the subcortex of the anteromedial superior cerebellum, the PSTT approach is recommended as an optimal surgical route. Scrupulous evaluation using preoperative neuroimaging is crucial when deciding on the surgical approach.
\end{abstract}

Key words: anteromedial superior cerebellar tumor, posterior subtemporal transtentorial approach, three-dimensional anisotropy contrast magnetic resonance axonography, tumor removal, vein of Labbé

\section{Introduction}

Various surgical routes have been described to access tumors located in the anteromedial superior cerebellum, including the lateral supracerebellar infratentorial (LSI), ${ }^{7)}$ occipital transtentorial (OTT), ${ }^{5,6)}$ and posterior subtemporal transtentorial (PSTT) approaches. ${ }^{1,3,4)}$ These surgical routes have been recognized as suitable for tumor resection in this region, but controversy remains as to which of these surgical routes is best when the superior pole of the tumor presents at the cerebellar surface..$^{1,3-5,7)}$ Furthermore, which of these routes offers the optimal approach to

Received November 5, 2007; Accepted August 5, 2008 a tumor that is not present at the cerebellar surface but instead lies within the deep anteromedial superior cerebellum is a matter of contention. In our institute, anteromedial superior cerebellar tumor is defined as a tumor located in the anteromedial half of the cerebellar hemisphere and superior to the middle cerebellar peduncle. Anteromedial superior cerebellar tumor is very rare,,$^{1)}$ and we have surgically treated only 5 patients with anteromedial superior cerebellar tumor between 1998 and 2006 in our institute. In these patients, we selected the surgical approach by preoperative evaluation based on individual tumor properties and anatomy. Tumor in the present case did not present at the surface of the anteromedial superior cerebellum. A PSTT approach was selected due to tumor location and anatomical 
structures surrounding the tumor on preoperative neuroimaging evaluation. We report herein a case in which hemangioblastoma in the deep anteromedial superior cerebellum was successfully removed using the PSTT approach, and discuss the application and advantages of this approach.

\section{Case Report}

A 44-year-old woman presented with mild headaches and vertigo. On admission, neurological examination revealed no deficit other than horizontal nystagmus. All preoperative image analyses including routine magnetic resonance (MR) imaging and three-dimensional anisotropy contrast MR axonography (3DAC) using diffusion-weighted MR imaging were performed on a 3.0-T MR system (Signa VH/I; GE Medical Systems, Milwaukee, Wis., U.S.A.). On 3DAC, nerve fibers running left-right (x-axis), anterior-posterior (y-axis), and superior-inferior (z-axis) appear as green, blue, and red, respectively. Mixed colors indicate oblique orientation of nerve fibers.

$\mathrm{T}_{1}$-weighted $\mathrm{MR}$ imaging with gadolinium revealed a mass lesion in the left anteromedial superior cerebellum (Fig. 1). Vertebral angiography demonstrated a hypervascular mass fed from branch of the superior cerebellar artery (SCA) (Fig. 2). Preoperative diagnosis was hemangioblastoma. Venousphase vertebral angiography demonstrated insertion of the vein of Labbé into the transverse-sigmoid junction. On $\mathrm{T}_{1}$-weighted MR imaging with gadolinium, the tumor did not present at the cerebellar surface of the anteromedial superior cerebellum, but lay within the subcortex. Coronal view image demonstrated that depth of tumor location from the cere-

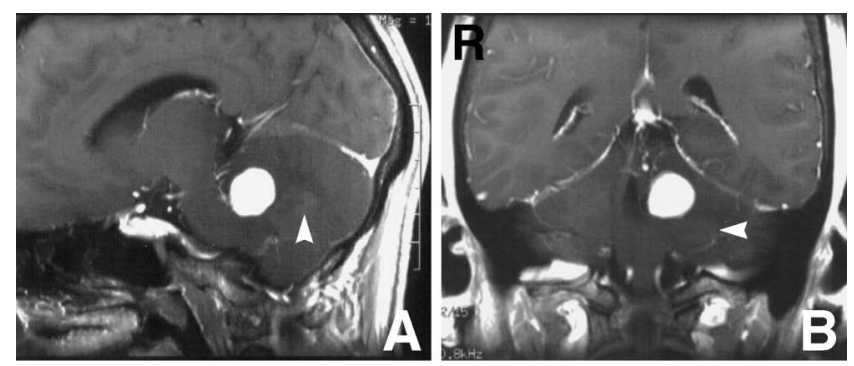

Fig. 1 Sagittal (A) and coronal (B) $T_{1}$-weighted magnetic resonance images with gadolinium revealing a homogeneously enhancing mass in the left anteromedial superior cerebellum adjacent to the pons and middle cerebellar peduncle (arrowhead). Coronal view demonstrates a small cyst medial to the mass. bellar surface was shorter than on sagittal view, and confirmed steep angulation of the tentorium. Sagittal 3DAC depicted the pontocerebellar tracts running radially from the middle cerebellar peduncle to the cerebellar cortex within the posterior quadrangular lobe posterosuperior to the white-colored tumor (Fig. 3A). These tracts seemed to intersect the surgical corridor for the LSI and OTT approaches. In contrast, coronal 3DAC revealed no integer fiber of the tectocerebellar tract within the anterior quadrangular lobe laterosuperior to the tumor (Fig. 3B).

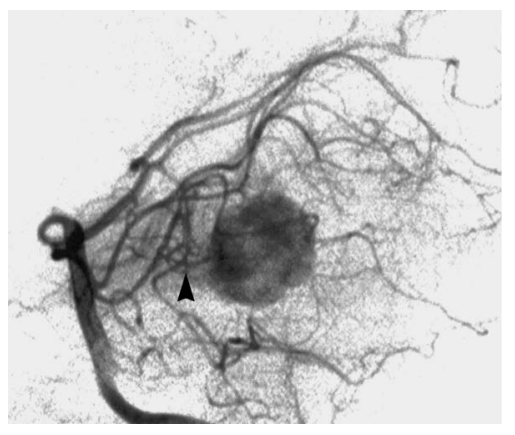

Fig. 2 Lateral left vertebral angiogram demonstrates branches of the superior cerebellar artery (arrowhead) entering directly into the tumor mass from anteriorly.

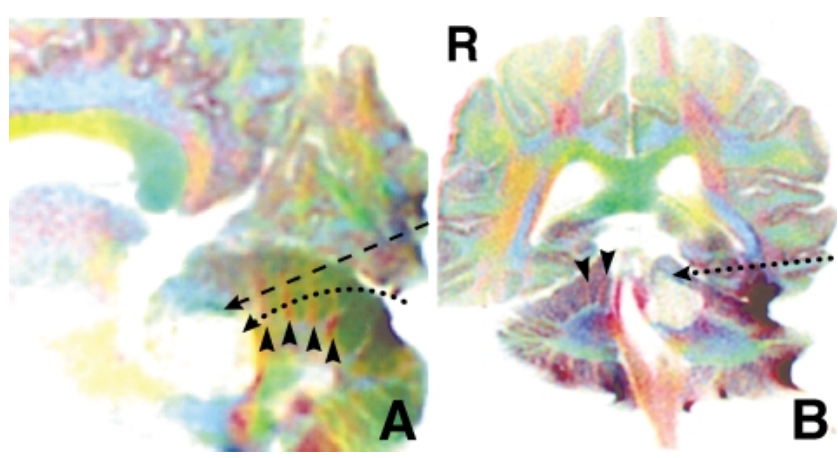

Fig. 3 A: Sagittal three-dimensional anisotropy contrast magnetic resonance axonogram clearly depicts the pontocerebellar tract (arrowheads) running radially and intersecting the surgical corridors of the lateral supracerebellar infratentorial (dotted line) and occipital transtentorial (dash line) approaches. B: Coronal three-dimensional anisotropy contrast magnetic resonance axonogram depicts the tectocerebellar tract within the subcortex of the contralateral side (arrowheads), but no fiber intersecting the surgical corridor of the posterior subtemporal transtentorial approach (dotted line) in the pathological side. 
We decided to use the PSTT approach for tumor removal in this case.

After initiation of general anesthesia, we placed a lumbar cerebrospinal fluid (CSF) drain and closed the catheter. In the supine position, the head of the patient was turned $80^{\circ}$ away from the vertical plane, and the vertex was tilted $30^{\circ}$ down to the floor so that the tentorium rose sheer from the horizontal plane. A question mark-shaped skin incision and square-shaped craniotomy were made at the region of presumed exposure of the vein of Labbé after inci-

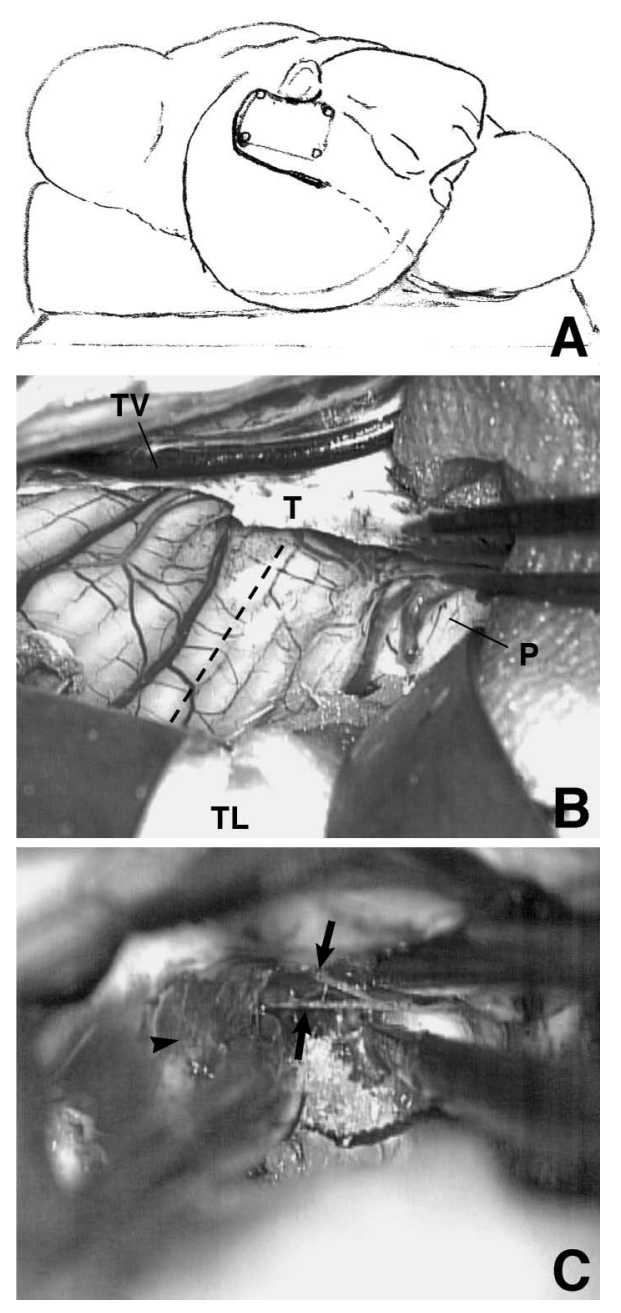

Fig. 4 Intraoperative images. A: A sketch of head fixation. Thick line, skin-incision line; dotted line, hair line. B: Surgical working field after incision of the tentorium. Dotted line, split line of a fissure in the anterior quadrangular lobule of the superior cerebellum; P, pons; T, tentorium; TL, temporal lobe; TV, temporal vein. C: Microsurgical view cutting the tumor feeding arteries during tumor removal. Arrows, tumor feeding arteries; arrowhead, tumor. sion of the dura mater (Fig. 4A). The lower temporal bone was then planed with a drill to reveal the temporal fossa. We did not make a horseshoe-shaped skin incision in the present case, because a turned muscle flap interrupted the surgical corridor during the subtemporal approach. The dura mater was incised in a U-shaped flap, and was turned and tacked to the caudal side. After dural incision, the lumbar drainage catheter was opened and CSF drained until sufficient relaxation of the temporal lobe was obtained. The arachnoid mater surrounding the vein of Labbe was dissected to ensure flexibility of this vein. Before retraction of the temporal lobe, we inserted 4 $\times 2 \times 1$-cm pieces of gelatin sponge at both ends between the base of the temporal lobe and the tentorium. These devices, CSF drainage, and gravity acting on the temporal lobe resulting from optimal head position allowed a minimal retraction of the temporal lobe. We gently retracted the temporal lobe and approached the tentorium. After confirmation of the trochlear nerve located under the tentorial edge, the tentorium was dissected straight to the free edge parallel with the superior petrosal sinus (Fig. 4B). The fissure of the anterior quadrangular lobe was split in the superior cerebellum, reaching to the reddish colored tumor. Although branches of the SCA as the tumor feeding artery directly entered to the tumor from anteriorly within the subcortex, we were able to find and easily coagulate these branches using a bipolar forceps due to an operative field enabling visualization of the proximal side of the SCA (Fig. 4C). After cutting the feeding vessels surrounding the tumor, tumor was separated from cerebellar tissue and completely removed (Fig. 5). Pieces of gelatin sponge inserted into both ends between the base of the temporal lobe and tentorium were removed before dura suturing. Histological diagnosis was hemangioblastoma.

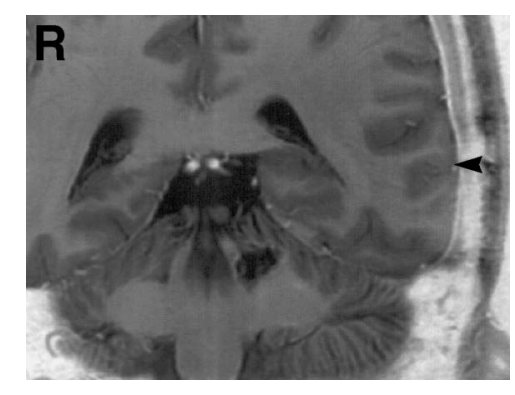

Fig. 5 Postoperative short inversion-time inversion recovery magnetic resonance image showing the tumor was completely removed without any injury to the temporal lobe or vein of Labbé (arrowhead). 
Postoperatively, the patient presented with vertigo and horizontal nystagmus, but achieved full recovery by 1 month postoperatively.

\section{Discussion}

The main surgical routes to access the anteromedial region of the superior cerebellum are the LSI, OTT, and PSTT approaches. We performed preoperative examinations to determine the best route for the present case. As the LSI approach was originally defined as a route to access the pineal region and midbrain through the supracerebellar infratentorial space ${ }^{8,11,12)}$ LSI is applied for access to the surface of the superior cerebellum on the way to the surgical corridor to the pineal region and midbrain. For the present case, however, we concluded that use of LSI approach would be difficult as substantial retraction of the cerebellum would be required to visualize the inferior pole of the tumor (Fig. 3A). OTT and PSTT approaches provide the advantage of direct tumor visualization from superior and lateral sides, respectively. Some reports have documented that little separates these approaches in cases with tumor presenting at the surface of the anteromedial superior cerebellum. ${ }^{4,5,7)}$ In the present case, the OTT approach would have required a long surgical corridor (approximately $5.5 \mathrm{~cm}$ ) and a thick cerebellar split. Furthermore, 3DAC demonstrated the pontocerebellar tract running radially within the posterior quadrangular lobe, which seemed to intersect the surgical corridor for the LSI and OTT approaches (Fig. 3A). The pontocerebellar tract comprises connective afferent fibers carrying information on intended movements from the cerebral cortex to the cerebellar cortex. ${ }^{13)}$ Interruption of this tract causes impairments to coordination and locomotion. ${ }^{10)}$ We were worried that the pontocerebellar tract would be damaged if an OTT or LSI approach was used. In contrast, the PSTT approach was expected to provide a shorter surgical corridor (approximately $4.5 \mathrm{~cm}$ ), and reduced split of the cerebellar cortex compared to the OTT approach. Finding of no integer fiber of the tectocerebellar tract within the anterior quadrangular lobe situated laterosuperior to the tumor on 3DAC also allowed us to choose the PSTT approach (Fig. 3B).

Ammerman et al. ${ }^{1)}$ documented 3 cases in which anteromedial superior cerebellar tumors were completely removed using the PSTT approach. In that report, anatomical features of the tumor and surrounding structures leading to amenable resection of tumor via this approach were: 1) presence of superior pole of the tumor at the cerebellar surface; 2) steep angulation of the tentorium; and 3) insertion of the vein of Labbe posterior to the transverse-sigmoid junction. Tumor in the present case did not present at the cerebellar surface, but lay within the subcortex. However, we successfully removed tumor and no complications arose. Preoperative 3DAC clearly depicted cerebellar nerve fibers adjacent to the tumor, thus helping in selection of the surgical route. In the present case, steep angulation of the tentorium provided almost direct perpendicular visualization of the tentorium and cerebellar surface. Our operative findings agreed with the statement of Ammerman et al. Risks during a PSTT approach include injuries to the temporal lobe and the vein of Labbé due to brain retraction. ${ }^{1-4,7)}$ Insertion of the vein of Labbe posterior to the transverse-sigmoid junction is thus desirable. Lonser et al. ${ }^{4)}$ recommended precluding this approach or using well-described techniques to avoid excessive stretch on the vein of Labbe if insertion occurs more anteriorly. In the present case, the vein of Labbé inserted into the transverse-sigmoid junction, but we were able to safely remove the tumor without injuring the vein of Labbe thanks to standard techniques such as optimal head position, CSF drainage, and detachment of the vein of Labbe from the cortex.9) Gelatin sponge was also inserted at both ends between the temporal lobe base and tentorium. This is useful for every operation requiring substantial retraction of the brain, such as subtemporal or interhemispheric approaches. However, we consider that use of the PSTT approach should be precluded if the insertion point is more anterior to the transverse-sigmoid junction.

Finally, the PSTT approach also facilitated isolation of tumor feeding vessels during surgery in this case. The PSTT approach provided an operative field with visualization of the proximal side of the SCA, allowing easy separation and coagulation of branches of the SCA entering to the tumor from the front within the cerebellar subcortex (Fig. 4C). In surgery for hemangioblastoma, complete cutting of tumor feeding arteries before tumor removal is very important to avoid intraoperative hemorrhage from the tumor. Isolating tumor feeding vessels in this case might have been difficult with LSI or OTT approaches, as these provide operative views of the tumor from posteriorly.

Although the present article is a case report, we gained the distinct impression that the PSTT approach offers a suitable route to tumors lying within the anteromedial superior cerebellum. Use of the PSTT approach for this region requires careful patient selection, needing shorter length of the surgical corridor, reduced cerebellar split, steep angulation of the tentorium, and preservation of nerve 
fibers surrounding tumor compared to other approaches. Estimation of these conditions using preoperative neuroimaging is very important when selecting the approach. The present case suggests injury of the vein of Labbe can be avoided with judicious application of various techniques, if the vein of Labbe inserts into the transverse-sigmoid junction. Further study of the PSTT approach in a limited group of patients with deep anteromedial superior cerebellar tumor is required to confirm the optimal route for access to this region.

\section{Acknowledgment}

This work was supported by the Advanced Medical Science Center at Iwate Medical University, Japan.

\section{References}

1) Ammerman JM, Lonser RR, Oldfield EH: Posterior subtemporal transtentorial approach to intraparenchymal lesions of the anteromedial region of the superior cerebellum. J Neurosurg 103: 783-788, 2005

2) Heros RC: Brain resection for exposure of deep extracerebral and paraventricular lesions. Surg Neurol 34: 188-195, 1990

3) Heros RC: Transtentorial approach. J Neurosurg 103: 776-777, 2005

4) Lonser RR, Ammerman JM, Oldfield EH: Subtemporal transtentorial approach. J Neurosurg 104: 855-856, 2006

5) Pompili A, Pace A, Occhipinti E: Subtemporal trans- tentorial approach. J Neurosurg 104: 854-855, 2006

6) Shirane R, Kumabe T, Yoshida Y, Su CC, Jokura H, Umezawa K, Yoshimoto T: Surgical treatment of posterior fossa tumors via the occipital transtentorial approach: evaluation of operative safety and results in 14 patients with anterosuperior cerebellar tumors. J Neurosurg 94: 927-935, 2001

7) Spetzler RF: Subtemporal transtentorial approach. J Neurosurg 104: 854, 2006

8) Stein BM: The infratentorial supracerebellar approach to pineal lesions. J Neurosurg 35: 197-202, 1971

9) Sugita K, Kobayashi S, Yokoo A: Preservation of large bridging veins during brain retraction. Technical note. J Neurosurg 57: 856-858, 1982

10) Terry JB, Rosenberg RN: Frontal lobe ataxia. Surg Neurol 44: 583-588, 1995

11) Vishteh AG, David CA, Marciano FF, Coscarella E, Spetzler RF: Extreme lateral supracerebellar infratentorial approach to the posterolateral mesencephalon: technique and clinical experience. Neurosurgery 46: 384-388, 2000

12) Yamamoto I: Pineal region tumor: surgical anatomy and approach. J Neurooncol 54: 263-275, 2001

13) Yaşargil MG: Microneurosurgery IV A. Stuttgart, New York, Georg Thieme Verlag, 1993, pp 80-83

Address reprint requests to: Takaaki Beppu, M.D., Department of Neurosurgery, Iwate Medical University, 19-1 Uchimaru, Morioka 020-8505, Japan.

e-mail: tbeppu@iwate-med.ac.jp 